\title{
Commentary: Deficient Inhibition in Alcohol-Dependence: Let's Consider the Role of the Motor System!
}

\author{
Lanjun Zhou, Bin Zhan, Weiqi He and Wenbo Luo* \\ Research Center of Brain and Cognitive Neuroscience, Liaoning Normal University, Dalian, China
}

Keywords: transcranial magnetic stimulation, alcohol dependence, motor system, inhibitory control, motor evoked potentials, action preparation

\section{A Commentary on}

Deficient Inhibition in Alcohol-Dependence: Let's Consider the Role of the Motor System!

by Quoilin, C., Wilhelm, E., Maurage, P., de Timary, P., and Duque, J. (2018). Neuropsychopharmacology 43, 1851-1858. doi: 10.1038/s41386-018-0074-0

OPEN ACCESS

Edited by:

Liana Fattore,

Italian National Research Council

(CNR), Italy

Reviewed by:

Nahian Chowdhury,

University of Sydney, Australia Primavera Alessandra Spagnolo, National Institutes of Health (NIH),

United States

*Correspondence:

Wenbo Luo

luowb@/nnu.edu.cn

Specialty section:

This article was submitted to Neural Technology,

a section of the journal

Frontiers in Neuroscience

Received: 20 April 2019 Accepted: 05 August 2019 Published: 20 August 2019

Citation:

Zhou L, Zhan B. He W and Luo W (2019) Commentary: Deficient Inhibition in Alcohol-Dependence: Let's Consider the Role of the Motor System! Front. Neurosci. 13:876. doi: 10.3389/fnins.2019.00876
Inhibitory control has a significant capacity to abort undesirable or inappropriate responses (Logan et al., 2014). As a part of executive functioning, it plays an important role in goal-directed behaviors (Luna et al., 2015). Previous literature has demonstrated that heavy drinking is related to higher impulsivity, including a reduced response inhibition (Ahmadi et al., 2013). Abstinent patients with alcohol dependence $(\mathrm{AD})$ have shown impairments in response inhibition, which could increase alcohol-elicited craving (Papachristou et al., 2012), contributing to compulsive drug-seeking behaviors and enhanced relapse risk (Dalley et al., 2011).

Substantial evidence has indicated that inhibitory control depends mainly on prefrontal areas (Berlin et al., 2004; Trantham-Davidson and Chandler, 2015; Klenowski, 2018). However, the motor system may have an impact on inhibitory control. Transcranial magnetic stimulation (TMS) studies have revealed a relationship between the corticospinal excitability changes and motor inhibition in the motor system (Duque et al., 2017); therefore, motor system excitability may be suppressed during response inhibition. Research on teenagers who suffered from heavy prenatal alcohol exposure recruited less primary motor areas in an easy inhibition response task when compared with control individuals (Ware et al., 2015). This highlights the influence of motor cortex on inhibitory function.

Single pulse TMS of the primary motor cortex (M1) can measure the extent of motor inhibition, reflected by motor-evoked potentials (MEPs) (Beck et al., 2008). MEPs decrease before a motor response, which corresponds with the results showing that the motor system is inhibited during the preparation of action (Greenhouse et al., 2015a). Although impairments in inhibitory control are found in individuals with addictive behaviors, it remains unclear whether the response inhibition is modulated by motor system in alcohol use disorders during action preparation.

This hypothesis is addressed in a recent paper published in the Neuropsychopharmacology (Quoilin et al., 2018). In this study, the authors explored the role of motor system in inhibitory control, behavioral inhibition, and relapse in patients with AD. Their main approaches involved the collection of demographical data, including the Spielberger State Trait Anxiety Inventory, Beck Depression Inventory, and UPPS Impulsive Behavior scale, behavior tests, and a TMS procedure. Quoilin et al. hypothesized that patients with $\mathrm{AD}$ had reduced neural motor inhibition during action preparation. To test this hypothesis, they utilized a $115 \%$ resting motor threshold of singlepulse TMS over the non-dominant or dominant hand area of the M1 to elicit MEPs corresponding to finger muscles during an instructed-delay choice reaction time (RT) task. 
The results were consistent with previous studies (Henges and Marczinski, 2012; Greenhouse et al., 2015b). Firstly, patients with $\mathrm{AD}$ showed a lack of behavioral inhibition and higher scores in trait impulsivity compared with the control group. Second, although suppression of MEPs was observed in the delay period relative to baseline in all participants for the forthcoming movement, the suppression for patients with $\mathrm{AD}$ was weaker than the controls (Greenhouse et al., 2015b; Lebon et al., 2016). Besides, both behavioral inhibition and suppression of MEPs were not associated with trait impulsivity. This supports the independence of diverse types of impulsivity, including impulsive choice (also referred to discounting of delayed rewards), impulsive action (response or motor inhibition), and impulsive personality traits (MacKillop et al., 2016). Delay discounting (but not response inhibition) is different between heavy drinking smokers and heavy drinkers only or smokers only (Moallem and Ray, 2012). Hence, the distinction of response inhibition during action preparation in different addictive groups should be addressed in the future. Finally, the authors extended these results by illustrating that the loss of inhibition was only found in relapsing patients after a year, while the persistent abstainers displayed comparable inhibitory control to healthy group in the neural and behavioral measurements. That is, a stronger disinhibition in patients indicated a higher probability of relapse. Hence, we can classify patients with $\mathrm{AD}$, those prone to relapse and those who remain abstinent, and efficiently intervene through cognitive training and TMS technology to enhance their inhibitory control (Kohl et al., 2019), which may promote better rehabilitation. In addition, this is the first study to show that the paucity of inhibitory function is modulated by the motor system in patients with $\mathrm{AD}$, which provides a new target of TMS for the future treatments.

Nonetheless, this study highlights some limitations. Addiction is characterized by alterations in multiple regions and brain circuits, but the effect of these related regions is not considered in the paper. In fact, the right frontotemporal (Gan et al., 2014), medial prefrontal cortex (Klenowski, 2018), and striatum (Cheng et al., 2017) are involved in inhibitory control in relevant alcohol studies; weaker functional connectivity between the frontal cortex and striatum has been found when serious alcohol users perform the response inhibition tasks (Courtney et al., 2013). Thus whether the inhibitory mechanism of M1 during action preparation depends on other brain areas should be investigated in the future. For instance, functional connectivity between M1 and other brain networks, such as the pre-supplementary motor area, or right inferior prefrontal cortex, is vital to successful inhibitory control (Duann et al., 2009). Some investigators have found that these prefrontal areas transmit information to the M1 to inhibit premature behaviors by an oscillatory beta rhythm (Picazio et al., 2014); the mechanism underlying these connections in individuals with addiction remains unknown. Combining TMS with fMRI, researchers can observe the changes of functional connectivity between M1 and other brain areas and help improve inhibitory control in patients with $\mathrm{AD}$ in the future. As preliminary studies, smokers can decrease their craving by real-time fMRI neurofeedback (Hartwell et al., 2016). Same technology can be applied to help the patients with $\mathrm{AD}$ to regulate their inhibitory control.
Besides, additional difference between persistent abstainers and relapsed patients remains unknown. For example, it is not clear whether alcohol consumption and dependence time between these two kinds of patients have significant difference. Previous research has shown that higher alcohol consumption is associated with less total brain volume (Paul et al., 2008) and neurocognitive impairment in multiple regions (Woods et al., 2016). Therefore, alcohol use history should be defined as covariate in the future. Although multiple cognitive functions are impaired in various brain regions for patients with $\mathrm{AD}$, longer alcohol withdrawal period promotes functional recovery (Kopera et al., 2012). Thus, the alterations of relevant brain networks in different abstinent duration should be further investigated.

Finally, M1 mechanism of inhibitory control is also revealed by paired-pulse TMS, including short- (SICI) and long-interval intracortical inhibition (LICI), involving gamma-aminobutyric acid A (GABAA) and gamma-aminobutyric acid B (GABAB), respectively. Previous research has found that reduced LICI of dorsolateral prefrontal cortex, no difference for SICI of motor cortex in $\mathrm{AD}$ patients post-detoxification compared with controls (Naim-Feil et al., 2016). However, the changes on LICI and SICI of $\mathrm{M} 1$ in patients with $\mathrm{AD}$ during action preparation need to be further explored. For healthy individuals, LICI is reduced during the whole response inhibition task, while reduced SICI is only found in informative cues (Cirillo et al., 2018); Individuals with better improvement in motor training show a reduction in GABAergic release in movement preparation (Dupont-Hadwen et al., 2019). Hence, Combining TMS and motor training, researchers should further investigate how to improve inhibitory control in patients with $\mathrm{AD}$.

In conclusion, the current work explored the different capacities for inhibition between patients with $\mathrm{AD}$ and healthy controls, including neural motor, behavior, and trait impulsivity. These researchers discovered that patients with $\mathrm{AD}$ had reduced motor cortex excitability and higher trait impulsivity compared with the controls. In addition, they reported a dysfunction in the neural inhibitory ability of patients with $\mathrm{AD}$ during movement preparation, especially in patients who had relapsed one year later. These findings have revealed the importance of inhibitory processes in forthcoming actions to healthy individuals. These data suggest that improved inhibitory control plays a significant role in preventing a relapse in serious alcoholism. Using TMS over the related motor cortex to modify inhibitory processes may be a prospective treatment for patients with addiction (Dupont-Hadwen et al., 2019).

\section{AUTHOR CONTRIBUTIONS}

LZ, WL, and WH conceived the idea, revised all the literature, and wrote the manuscript. BZ read and revised the manuscript. $\mathrm{WH}$ and WL contributed to the revision of the manuscript. All authors read and approved the submitted version.

\section{FUNDING}

This work was supported by the National Natural Science Foundation of China (Grant Nos. 31871106 and 81601166 ). 


\section{REFERENCES}

Ahmadi, A., Pearlson, G. D., Meda, S. A., Dager, A., Potenza, M. N., Rosen, R., et al. (2013). Influence of alcohol use on neural response to go/no-go task in college drinkers. Neuropsychopharmacology 38, 2197-2208. doi: 10.1038/npp.2013.119

Beck, S., Richardson, S. P., Shamim, E. A., Dang, N., Schubert, M., and Hallett, M. (2008). Short intracortical and surround inhibition are selectively reduced during movement initiation in focal hand dystonia. J. Neurosci. 28, 10363-10369. doi: 10.1523/JNEUROSCI.3564-08.2008

Berlin, H. A., Rolls, E. T., and Kischka, U. (2004). Impulsivity, time perception, emotion and reinforcement sensitivity in patients with orbitofrontal cortex lesions. Brain 127, 1108-1126. doi: 10.1093/brain/awh135

Cheng, Y., Huang, C. C. Y., Ma, T., Wei, X., Wang, X., Lu, J., et al. (2017). Distinct synaptic strengthening of the striatal direct and indirect pathways drives alcohol consumption. Biol. Psychiatry 81, 918-929. doi: 10.1016/j.biopsych.2016.05.016

Cirillo, J., Cowie, M. J., MacDonald, H. J., and Byblow, W. D. (2018). Response inhibition activates distinct motor cortical inhibitory processes. J. Neurophysiol. 119, 877-886. doi: 10.1152/jn.00784.2017

Courtney, K. E., Ghahremani, D. G., and Ray, L. A. (2013). Fronto-striatal functional connectivity during response inhibition in alcohol dependence. Addict. Biol. 18, 593-604. doi: 10.1111/adb.12013

Dalley, J. W., Everitt, B. J., and Robbins, T. W. (2011). Impulsivity, compulsivity, and top-down cognitive control. Neuron 69, 680-694. doi: 10.1016/j.neuron.2011.01.020

Duann, J.-R., Ide, J. S., Luo, X., and Li, C.-S. R. (2009). Functional connectivity delineates distinct roles of the inferior frontal cortex and presupplementary motor area in stop signal inhibition. J. Neurosci. 29, 10171-10179. doi: 10.1523/JNEUROSCI.1300-09.2009

Dupont-Hadwen, J., Bestmann, S., and Stagg, C. J. (2019). Motor training modulates intracortical inhibitory dynamics in motor cortex during movement preparation. Brain Stimul. 12, 300-308. doi: 10.1016/j.brs.2018. 11.002

Duque, J., Greenhouse, I., Labruna, L., and Ivry, R. B. (2017). Physiological markers of motor inhibition during human behavior. Trends Neurosci. 40, 219-236. doi: 10.1016/j.tins.2017.02.006

Gan, G., Guevara, A., Marxen, M., Neumann, M., Juenger, E., Kobiella, A., et al. (2014). Alcohol-induced impairment of inhibitory control is linked to attenuated brain responses in right fronto-temporal cortex. Biol. Psychiatry 76, 698-707. doi: 10.1016/j.biopsych.2013.12.017

Greenhouse, I., Saks, D., Hoang, T., and Ivry, R. B. (2015a). Inhibition during response preparation is sensitive to response complexity. J. Neurophysiol. 113, 2792-2800. doi: 10.1152/jn.00999.2014

Greenhouse, I., Sias, A., Labruna, L., and Ivry, R. B. (2015b). Nonspecific inhibition of the motor system during response preparation. J. Neurosci. 35, 10675-10684. doi: 10.1523/JNEUROSCI.1436-15.2015

Hartwell, K. J., Hanlon, C. A., Li, X., Borckardt, J. J., Canterberry, M., Prisciandaro, J. J., et al. (2016). Individualized real-time fmri neurofeedback to attenuate craving in nicotine-dependent smokers. J. Psychiatry Neurosci. 41, 48-55. doi: $10.1503 /$ ipn. 140200

Henges, A. L., and Marczinski, C. A. (2012). Impulsivity and alcohol consumption in young social drinkers. Addict. Behav. 37, 217-220. doi: 10.1016/j.addbeh.2011.09.013

Klenowski, P. M. (2018). Emerging role for the medial prefrontal cortex in alcohol-seeking behaviors. Addict. Behav. 77, 102-106. doi: 10.1016/j.addbeh.2017.09.024

Kohl, S., Hannah, R., Rocchi, L., Nord, C. L., Rothwell, J., and Voon, V. (2019). Cortical paired associative stimulation influences response inhibition: cortico-cortical and cortico-subcortical networks. Biol. Psychiatry 85, 355-363. doi: 10.1016/j.biopsych.2018.03.009
Kopera, M., Wojnar, M., Brower, K., Glass, J., Nowosad, I., Gmaj, B., et al. (2012). Cognitive functions in abstinent alcohol-dependent patients. Alcohol 46, 665-671. doi: 10.1016/j.alcohol.2012.04.005

Lebon, F., Greenhouse, I., Labruna, L., Vanderschelden, B., Papaxanthis, C., and Ivry, R. B. (2016). Influence of delay period duration on inhibitory processes for response preparation. Cereb. Cortex 26, 2461-2470. doi: 10.1093/cercor/bhv069

Logan, G. D., Van Zandt, T., Verbruggen, F., and Wagenmakers, E.-J. (2014). On the ability to inhibit thought and action: general and special theories of an act of control. Psychol. Rev. 121, 66-95. doi: 10.1037/a0035230

Luna, B., Marek, S., Larsen, B., Tervo-Clemmens, B., and Chahal, R. (2015). An integrative model of the maturation of cognitive control. Ann. Rev. Neurosci. 38, 151-170. doi: 10.1146/annurev-neuro-071714-034054

MacKillop, J., Weafer, J., Gray, J. C., Oshri, A., Palmer, A., and de Wit, H. (2016). The latent structure of impulsivity: impulsive choice, impulsive action, and impulsive personality traits. Psychopharmacology 233, 3361-3370. doi: 10.1007/s00213-016-4372-0

Moallem, N. R., and Ray, L. A. (2012). Dimensions of impulsivity among heavy drinkers, smokers, and heavy drinking smokers: singular and combined effects. Addict. Behav. 37, 871-874. doi: 10.1016/j.addbeh.2012.03.002

Naim-Feil, J., Bradshaw, J. L., Rogasch, N. C., Daskalakis, Z. J., Sheppard, D. M., Lubman, D. I., et al. (2016). Cortical inhibition within motor and frontal regions in alcohol dependence post-detoxification: a pilot tms-eeg study. World J. Biol. Psychiatry 17, 547-556. doi: 10.3109/15622975.2015.1066512

Papachristou, H., Nederkoorn, C., Havermans, R., van der Horst, M., and Jansen, A. (2012). Can't stop the craving: the effect of impulsivity on cue-elicited craving for alcohol in heavy and light social drinkers. Psychopharmacology 219, 511-518. doi: 10.1007/s00213-011-2240-5

Paul, C. A., Au, R., Fredman, L., Massaro, J. M., Seshadri, S., DeCarli, C., et al. (2008). Association of alcohol consumption with brain volume in the framingham study. Arch. Neurol. 65, 1363-1367. doi: 10.1001/archneur.65.10.1363

Picazio, S., Veniero, D., Ponzo, V., Caltagirone, C., Gross, J., Thut, G., et al. (2014). Prefrontal control over motor cortex cycles at beta frequency during movement inhibition. Curr. Biol. 24, 2940-2945. doi: 10.1016/j.cub.2014.10.043

Quoilin, C., Wilhelm, E., Maurage, P., de Timary, P., and Duque, J. (2018). Deficient inhibition in alcohol-dependence: let's consider the role of the motor system! Neuropsychopharmacology 43, 1851-1858. doi: 10.1038/s41386-018-0074-0

Trantham-Davidson, H., and Chandler, L. J. (2015). Alcohol-induced alterations in dopamine modulation of prefrontal activity. Alcohol 49, 773-779. doi: 10.1016/j.alcohol.2015.09.001

Ware, A. L., Infante, M. A., O’Brien, J. W., Tapert, S. F., Jones, K. L., Riley, E. P., et al. (2015). An fmri study of behavioral response inhibition in adolescents with and without histories of heavy prenatal alcohol exposure. Behav. Brain Res. 278, 137-146. doi: 10.1016/j.bbr.2014.09.037

Woods, A. J., Porges, E. C., Bryant, V. E., Seider, T., Gongvatana, A., Kahler, C. W., et al. (2016). Current heavy alcohol consumption is associated with greater cognitive impairment in older adults. Alcoholism 40, 2435-2444. doi: 10.1111/acer.13211

Conflict of Interest Statement: The authors declare that the research was conducted in the absence of any commercial or financial relationships that could be construed as a potential conflict of interest.

Copyright (c) 2019 Zhou, Zhan, He and Luo. This is an open-access article distributed under the terms of the Creative Commons Attribution License (CC BY). The use, distribution or reproduction in other forums is permitted, provided the original author(s) and the copyright owner(s) are credited and that the original publication in this journal is cited, in accordance with accepted academic practice. No use, distribution or reproduction is permitted which does not comply with these terms. 